\title{
APERTURA COMERCIAL Y TRANSFORMACIONES EN EL PAPEL DEL ESTADO EN LA ECONOMÍA EN AMÉRICA LATINA, 1970-2014
}

\author{
Rodrigo Cuevas \\ Universidad de Chile \\ rodrigocuevas@uchile.cl
}

\section{RESUMEN}

El artículo analiza el impacto de la inserción en el comercio internacional sobre las transformaciones en el rol del Estado en la economía en 20 países latinoamericanos entre 1970 y 2014. Los datos fueron analizados a partir del Times Series Cross Section. Los resultados muestran la persistencia de una serie de patrones en la estructura económica de la región, tales como la importancia de los recursos naturales en los ingresos del Estado. Asimismo, el artículo muestra la importancia de los acuerdos internacionales que liberalizan la política comercial sobre el reforzamiento de la evolución en el rol económico del Estado.

Palabras claves: Apertura comercial, política comercial, Reforma del Estado, América Latina.

* El presente artículo forma parte del proyecto "Programa de Estímulo a la Excelencia Institucional 2014”, del Instituto de Estudios Internacionales de la Universidad de Chile, de la Vicerrectoría de Investigación y Desarrollo de dicha casa de estudios. 


\title{
FREE TRADE AND TRANSFORMATIONS IN THE STATE ROLE IN LATIN AMERICAN ECONOMY, 1970-2014
}

\begin{abstract}
This article analyzes the impact of the insertion on international trade over the changes experimented by the State's role in political economy, in 20 Latin American countries between 1970 and 2014. Data sets were treated by and studied through Time-Series Cross Section analyses. The results show the persistence over time of patterns of Latin America's economic structure, such as the importance of natural resources in the state's revenue. Besides, the article shows the importance of international agreements that liberalize trade policy over the reinforcement of the evolution of the State's economic role.
\end{abstract}

Keywords: Free Trade, Trade Policy, State Reform, Latin America 


\section{INTRODUCCIÓN Y ANTECEDENTES}

El presente trabajo busca identificar variables, relacionadas con la apertura del comercio, que influyen en la evolución hacia un Estado con menor participación activa en la economía, a partir del análisis de 20 países ${ }^{1}$ de América Latina en un periodo entre 1970 y 2014. El estudio de la relación del Estado con el comercio se explica,, por un lado, porque el comercio da cuenta de la vinculación con los mercados externos, lo que permite dimensionar una orientación en el largo plazo de su respectivo ordenamiento económico. Por otro, y en especial a partir del papel que a partir de la década de 1990, importa estudiar el efecto de determinados acuerdos comerciales, que avanzan hacia la instalación y permanencia en el tiempo de un determinado de modelo económico.

En ese sentido, ambas dimensiones dan cuenta de un proceso relacionado a partir de los efectos en la adopción de procesos de reformas económicas orientadas al mercado. Entre las definiciones de reformas orientadas al mercado, se toma como punto de partida la elaborada por Adam Przeworski, quien señala que ellas tienen que ver con maneras de asignar más eficientemente los recursos que permiten que el Estado sea financieramente solvente. La primera parte del término, tiene que ver con la (re)organización de mercados, desregulación de precios y disminución en la protección, mientras que en la segunda lo es respecto a cómo este actor logra una reducción de gasto público e incrementar su posición fiscal (Przeworski 1991: 136).

En este artículo se utilizará el término reforma orientada al mercado, pues se lo considera más útil que el concepto neoliberalismo. Si bien ambos términos se refieren a políticas que apuntan a la desregulación y la reducción del Estado, el neoliberalismo es un enfoque que refiere a transformaciones más amplias en el orden social, de las cuales este documento no pretende ni puede dar cuenta (Evans y Sewell 2013: 36-38), ni siquiera en el aspecto meramente económico (Crouch, 2009, 2011, 2013, Schmidt y Tatcher 2013, Streeck 2012).

1 Los países considerados fueron Argentina, Bolivia, Brasil, Chile, Colombia, Costa Rica, Cuba, República Dominicana, Ecuador, El Salvador, Guatemala, Honduras, Jamaica, México, Nicaragua, Panamá, Paraguay, Perú, Uruguay y Venezuela. 
Este artículo tampoco pretende estudiar el efecto de las condiciones previas en que este proceso se da (De Melo, Denizer, Gelb, y Tenev 2001), que son las circunstancias específicas para las cuales Przeworski planteó la definición, que corresponden a un período entre mediados de las décadas de 1980 y 1990. Estas circunstancias tienen que ver con un proceso simultáneo, que es el de democratización, donde el vínculo entre ambos tiene que cómo estas reformas se hacen en condiciones de cambio de régimen político o cómo afectan decisivamente la inauguración de democracias (Haggard y Kaufman 1997: 277). Entre las particularidades que presenta el caso latinoamericano, está el hecho que las reformas económicas son procesos de ajuste estructural y no un cambio total de sistema, como ocurrió en los países de Europa Central y Oriental que pertenecían a la zona de influencia de la Unión Soviética (Przeworski 1991: 140)

En este contexto, el presente trabajo se plantea estudiar, para el caso de los países considerados, la evolución en el proceso de transformación del Estado, en cuanto a actor económico, hacia un menor protagonismo, el que es asumido por el sector privado. Igualmente, se estudia cómo este se ve afectado por variables relacionadas con un proceso específico de inserción económica en los mercados internacionales, como la apertura del comercio.

Para el caso de América Latina, respecto al papel que estas variables, es posible distinguir dos líneas principales. La primera de ellas tiene relación con el rol que cumple el Estado en la definición de un modelo económico, más cerrado o abierto a los mercados internacionales. En ese sentido, una primera etapa tiene que ver con el papel que cumplía en un modelo sustitutivo de importaciones (ISI), donde se buscaba proteger a determinados sectores productivos de la competencia externa, aunque respecto al papel del comercio no se plantea per se como un modelo cerrado al mismo. En efecto, Raúl Prebisch, quien es reconocido como una de las principales referencias del modelo ISI, argumentaba que este debía ser un elemento propulsor del desarrollo productivo, siendo el problema en cómo América Latina, en su proceso de industrialización, era capaz de insertarse internacionalmente en los mercados de una manera distinta a la exportación de bienes primarios (Prebisch 1986: 480-481).

En ese sentido, el papel del Estado se definía en función al apoyo de un proceso de desarrollo económico con énfasis en la industrialización y en 
cómo la región podía mejorar sus términos de intercambio como efecto de este cambio en su modelo productivo. Ello derivó en que, hasta al menos parte importante de la década de 1980, la política comercial fue concebida en este como una pieza fundamental. (Ffrench-Davis 1973, Hirschman 1968, Love 1990, Palma 1978, Prebisch 1986, Urquidi 2005) no obstante ya a fines de la década de 1960, empezarán a surgir críticas respecto a su agotamiento (Baer 1972, Ffrench-Davis 1973).

Estos cuestionamientos pueden sintetizarse en dos perspectivas. Por un lado, en la insuficiencia de la política comercial para reducir la brecha respecto del centro (los países desarrollados), la que se habría acentuado hasta llegar a constituirse en una relación de dependencia ${ }^{2}$ (Cardoso y Falleto 1969, Love 1990). Por otro lado, se planteaba un giro hacia una mayor liberalización, debido a que el esquema ISI generó un sector productivo ineficiente, situación que se habría agudizado con la crisis de la deuda de en la década de 1980, que habría proporcionado el entorno preciso para este cambio (Urquidi 2005). En ambos enfoques, la apertura cumple un rol funcional a un modelo de desarrollo económico, en el cual el Estado tiene un rol determinado. Por un lado, como un actor económico mediante, por ejemplo, un rol empresarial, y por otro, el énfasis puesto más bien en su función (des)reguladora.

A partir de la mayor aceptación del segundo enfoque, la que genera el entorno para la adopción de reformas de mercado, el comercio cumple una función distinta a otras medidas que se incluyen en este tipo de transformaciones en el orden económico. Algunas de estas se planteaban ante una etapa crítica pero acotada en el tiempo, como lo era por ejemplo, el control de una hiperinflación o del déficit público. Es decir, una función de estabilización. En cambio, la apertura comercial corresponde a una fase distinta (liberalización), que se refiere más hacia una consolidación de las reformas en el tiempo, proceso que ocurriría mediante la profundización de la inserción económica en el exterior (Bresser Pereira, Maravall y Przeworski 1995: 12-14)

2 El debate sobre la Teoría de la Dependencia y sus alcances han sido objeto de un amplio debate que no alcanza a ser cubierto en el presente artículo, además de no ser un foco del mismo. Una síntesis al respecto puede encontrarse en (Love 1990, Palma 1978) 
En ese sentido, es interesante observar la evolución que adquiere el concepto política comercial, que da cuenta de la variación de los énfasis, y que se observa en el tránsito desde una idea de commercial policy a otra de trade policy. Si bien una traducción literal indicaría ambos términos como sinónimos, ello es un error, porque en el primer caso se refiere al énfasis en regular la protección del comercio, mientras que en el segundo, el foco es puesto está puesto en la liberalización.

Ello se muestra en el tránsito desde el Acuerdo General de Aranceles y Servicios $(\mathrm{GATT})^{3}$, donde el énfasis está puesto en el primer término, hacia la creación de la Organización Mundial del Comercio (OMC) en 1995, en el cual el foco pasa a ser una aproximación del tipo trade policy, la que se refuerza la actual etapa, donde, junto al sistema multilateral de comercio (OMC), coexiste un fenómeno de proliferación de acuerdos comerciales regionales (ACR) (Goode 2007, OMC 2011)

Junto con una evolución de los mecanismos institucionales, también se constata un cambio en los énfasis de cada uno. En ese sentido, el cambio está en que el logro de una determinada apertura del comercio de bienes (mediante la reducción de aranceles a las importaciones), objetivo que se habría logrado en su mayoría tras la creación de la OMC, deja de ser relevante. Ello, porque entre los requisitos para ser miembro de esta organización está la consolidación ${ }^{4}$ de sus aranceles, que es básicamente el compromiso de no aumentarlos, lo que significó un punto de no retorno, en el cual las opciones de política en esta materia son reducción o mantención de los compromisos adquiridos.

3 El GATT es la sigla para el Acuerdo General de Comercio y Aranceles, que se comenzó a negociar multilateralmente en 1947 y cuya negociación culminó en 1994, con la creación de la Organización Mundial del Comercio (OMC) en 1995. Este acuerdo se negoció por etapas, a través de un sistema de rondas, cuya última fue la Ronda Uruguay (porque se inició en ese país en 1985) y que culminó en los acuerdos de Marrakech en 1994.

4 Consolidación arancelaria se refiere al máximo arancel aduanero que un país podría llegar a aplicar para proteger un determinado producto o sector. Se diferencia del arancel aplicado, en que este se define como el que efectivamente aplica en un momento dado. (Goode 2007). Estos son aranceles de Nación más Favorecida (NMF), es decir que se aplican a todos los países, salvo con aquellos con los que se cuenta con algún tipo de acuerdo comercial, que contemplan beneficios solo para las partes involucradas. (OMC, ITC y UNCTAD 2013) 
Si bien los acuerdos de libre comercio tienen compromisos arancelarios, especialmente después de 1990, el énfasis que tendrían sería el de apoyar la adopción de reformas de mercado, sea en la etapa de implementación o consolidar los procesos de este tipo en curso (Baccini y Urpelainen 2014, Gathii 2011, Mansfield y Milner 2012). Esta característica sería especialmente relevante cuando se da la presencia de un acuerdo (ACR) con la Unión Europea o Estados Unidos, pues implicaría la presencia más allá de la regulación arancelaria, apuntando a aspectos propios del funcionamiento de economías de libre mercado, (des)regulando ámbitos como la protección de inversiones, derechos de propiedad intelectual, o incluso de compras públicas (Allee y Elsig 2016, Anderson, Pelletier, Osei-Lah, y Müller 2011, Baccini, Dür, Elsig, y Milewicz 2011, Baccini y Urpelainen 2014, Dür, Baccini, y Elsig 2014, Elsig 2007, Henrik, Mavroidis, y Sapir 2009, Low, Elms, y Low 2012, Mansfield y Milner 2012, Rodrik 2000)

En ese sentido, el marco en el queque se situaría el estudio de la variable dependiente (la transformación del rol económico del Estado) es cómo ella se ve afectada en este proceso de cambio por una serie de variables que explicarían este proceso. En ese sentido, la hipótesis que guiaría este análisis es que, efectivamente, posibles predictores relacionadas con el comercio tienen un efecto sobre una disminución en el rol del Estado en la economía en el período 1970-2104. Se descarta un análisis a partir de variables que den cuenta del grado de adhesión a principios de la libertad económica, como los de la Fundación, dado que el número de observaciones se reduce, pues los datos están disponibles desde 1995. La sección que sigue a continuación, presenta la metodología utilizada (análisis Times Series Cross Section, TSCS) y de las variables utilizadas. En el tercer apartado se exponen los principales resultados y discusión de los mismos. Finalmente, se plantean las conclusiones y preguntas generadas a partir del presente trabajo.

\section{METODOLOGÍA Y VARIABLES CONSIDERADAS}

La base de datos utilizada se construyó con observaciones del tipo paísaño, debido a que un tratamiento basado exclusivamente por su unidad de referencia (país) presentaría el problema de trabajar con un bajo número de casos (20 países). Ello no sería recomendable, por un lado, porque determinar el efecto de determinadas variables predictoras respecto 
a una explicada es esencialmente una aproximación variable-oriented, en la cual se busca estimar el efecto de una o más variables dentro de una población de casos. El énfasis, por lo tanto, está puesto en esta y no en las particularidades de las unidades (Mahoney y Goertz 2006: 230). En ese sentido, el uso de técnicas de regresión daría resultados sesgados a partir del bajo número de casos.

Por ello, se opta por incrementar el número de observaciones (países), incorporando la presencia de una unidad de tiempo (años), transformándolas en una estructura del tipo país-año, que se define como una forma de datos en que se da la presencia de casos observados en varios años (Allison 2009: ix). El enfoque aplicado para trabajar en este tipo de datos es el de TSCS (Beck y Katz 1995: 634), que consiste en la aplicación de una combinación regresiones con la presencia de parámetros bajo un principio de mínimos cuadrados ordinarios (OLS), con la adaptación de los errores estándar para la aplicación de trabajo con datos de panel (Beck y Katz 1995: 635).

Su uso se justifica porque el tipo de observaciones consideradas requiere el uso de técnicas de multinivel, al considerar no solo el efecto temporal sino que la incorporación variables de contexto. El enfoque TSCS permite el uso de un modelo OLS, lo que facilita la interpretación de los parámetros y, al mismo tiempo, incorpora elementos que posibilita lidiar con problemas que se presentan en este tipo de estructura de datos, como el rompimiento de uno de los supuestos básicos del modelo OLS, que es la de autocorrelación en los términos de error en distintas observaciones (Plümper, Troeger, y Manow 2005: 329, Schroeder, Sjoquist, y Stephan 1986: 73).

Dentro de los tipos de regresiones que se pueden utilizar en un enfoque TSCS, manteniendo parámetros del tipo OLS, se encuentran el uso de efectos fijos (fixed effects o FE) o aleatorios (random effects). La principal diferencia es que el primero asigna a cada unidad su propio error, mientras que el segundo lo hace aleatoriamente (Allison 2009, Bell y Jones 2015, Plümper, Troeger, y Manow 2005). Entre las ventajas que presenta el uso de FE, está que este permite soslayar la condición que los valores no observados sean independientes entre sí, que es un problema que se presenta en esta estructura de datos (Allison 2009: 1-3), mientras que se le cuestiona la tendencia a enfocarse exclusivamente al análisis al interior de 
los grupos de control (whitin effects), obviando lo que ocurre al nivel más alto, es decir entre los mismos (between effects), estimación que sí puede realizarse con la aplicación de un modelo de RE (Bell y Jones 2015: 138139, Plümper, Troeger, y Manow 2005: 334). En ese sentido, el uso de fixed effects implica optar por un menor sesgo en los parámetros obtenidos con el costo de reducir la eficiencia de los mismos (Allison 2009: 3).

En el caso del presente artículo, se optó por utilizar el modelo de efectos fijos, siguiendo a Beck, para quien estudiar características institucionales de países, que en muchos casos son invariantes (es decir, cambian lentamente en el tiempo), haría recomendable la utilización de una regresión con efectos fijos (FE) (Beck 2008: 483).

La variable dependiente, el papel del Estado en la economía, se definió a partir del indicador State Ownership of the Economy (State) que mide el grado de presencia, a través del control y propiedad de activos por parte del Estado, en la economía. Este se genera en el marco del proyecto Varieties of Democracy (V-DEM) (Coppedge et al. 2016), y consiste en una variable originalmente construida en escala ordinal. Los valores cero indican una presencia casi absoluta del Estado, mientras los valores cuatro muestran una importancia mayoritaria del sector privado. Posteriormente, la variable fue transformada a una de tipo intervalar, a fin de permitir su uso en un análisis TSCS (Coppedge et al. 2016: 221-222). En relación a sus valores, en la base de datos presenta un promedio de 2,67, con una desviación estándar bastante elevada y totaliza un total de 886 observaciones.

\section{Tabla 1: Descripción de variable dependiente}

\begin{tabular}{cccccc} 
Variable & Promedio & $\begin{array}{c}\text { Desviación } \\
\text { estándar }\end{array}$ & Mínimo & Máximo & N \\
\hline State $(1970-2014)$ & 2,67 & 0,786 & 0,0441 & 3,714 & 886
\end{tabular}

La inclusión de las variables predictoras corresponde a su agrupación en tres categorías. La primera corresponde al efecto de medidas relacionadas con la introducción de reformas de mercado. La segunda considera patrones específicos de exportaciones por sector. La tercera incorpora la presencia de determinados acuerdos comerciales que habrían cumplido el propósito de consolidar procesos de reforma económica. 
En la primera categoría se incluyen variables como la reducción del gasto general del gobierno y del control de la inflación, que representaría el efecto de políticas de estabilización económica, que si bien son tomadas en sus inicios, tendría posteriormente sobre un mayor papel del sector privado en vez del Estado. Al mismo tiempo, una mayor importancia del comercio (suma de exportaciones e importaciones) en relación al Producto Interno Bruto (PIB), tendría relación con una dinámica de consolidación de estas reformas, al orientarse sus respectivas estructuras económicas hacia los mercados externos. Junto con estas reformas, en la transformación del Estado debería afectar tanto un incremento en el PIB como el nivel de riqueza del mismo país medido como PIB per cápita (medidas en dólares de USD). Para efectos de interpretación, estas dos variables fueron operacionalizadas a partir del logaritmo natural. Los datos fueron obtenidos de la base World Development Indicators del Banco Mundial (Banco Mundial 2014).

En la segunda categoría se incorporan indicadores que dan cuenta de la importancia de las materias primas en la economía y del desarrollo de un sector industrial. En primer lugar, la renta obtenida a partir de los recursos naturales, que es muy sensible a la demanda en los mercados internacionales, que, para el caso de América Latina, es un patrón que se mantiene en el tiempo. Ello se contrasta con el nivel de exportaciones del sector manufacturero, en el cual una mayor importancia del mismo indicaría un cambio en lo que fue la tendencia en los países latinoamericanos. En ese sentido, países con una mayor dependencia de los recursos naturales tendrían menores incentivos a desarrollar otros sectores (Banco Mundial 2014).

Asimismo, se incluyeron dos variables que buscan caracterizar procesos históricos de apertura hacia el comercio, a partir de la entrada en vigor del GATT y de un acuerdo de libre comercio con los Estados Unidos. En algunos casos, se ha identificado al GATT como un punto de inflexión a partir del cual se comienza a introducir procesos de reformas que reducen el papel del Estado en la economía (Murillo 2001, Urquidi 2005). En el caso de los acuerdos, estos involucran compromisos importantes en la reformas de aspectos regulatorios (Baccini y Urpelainen 2014, Dür, Baccini y Elsig 2014, Mansfield y Milner 2012). Los datos fueron obtenidos, para el caso de la membresía en el GATT, de la Organización de Estados Americanos (OEA 2015). Para el caso de los acuerdos de libre comercio 
con los Estados Unidos, los datos considerados fueron el año que entró en vigor ese acuerdo, los que se obtuvieron de la Organización Mundial del Comercio (OMC 2015).

\section{RESULTADOS}

El análisis se ejecutó por medio de la utilización de regresiones de efecto fijo, en lo que se presentan resultados sobre la variable dependiente (State), con modelos para cada una de las tres categorías consideradas. Un cuarto modelo considera la presencia de la totalidad de los predictores utilizados. En una primera etapa, se evaluó la pertinencia de utilizar un modelo de efectos fijos o uno aleatorio por medio del análisis del efecto de las variables macroeconómicas señaladas sobre esta transformación del Estado. Ello confirmaría o no las premisas expuestas en el párrafo anterior, que señalan la conveniencia del primero.

Esta definición permite acotar el análisis al interior de las entidades que conforman el panel (países) o, más bien, permitirían expandirlo hacia una comparación entre las mismas. Al mismo tiempo, dependiendo del modelo elegido, se aplican test destinados a evaluar la presencia de problemas asociados a la aplicación de este modelo, e introducir los ajustes pertinentes.

A partir de los resultados de la aplicación del test de Hausman ${ }^{5}$, se confirma como una mejor opción el modelo de fixed effects. Dentro de sus diagnósticos asociados, el resultado de la aplicación del test Pesaran CD señala que los términos de error no son independientes. Se presentan problemas de heterocedasticidad y autocorrelación de primer orden. Tomando en cuenta la revisión realizada y dada la estructura de datos, se recomienda la aplicación de regresiones con corrección de panel de los errores estándar (PCSE). Ello porque al asumir la existencia de términos de error heterocedásticos y de autocorrelación al interior (y entre) de las entidades del panel, controla estos efectos (StataCorp 2013: 287-294)

5 Se rechaza a un $\mathrm{p}<0.000$ la $\mathrm{H}_{0}$ que las diferencias en los coeficientes no son sistemáticas. Por lo tanto, se descartala pertinencia de utilizar un modelo de efectos aleatorios. 
La utilización de este método es recomendable porque permite la interpretación de los parámetros OLS, introduciendo la corrección de los términos de error de la manera señalada, recomendando su aplicación para un análisis TSCS, en especial cuando el número de unidades es superior al de los puntos de tiempo (Beck y Katz 1995: 640-641, 644) La técnica que se utiliza, por lo tanto, en los modelos corresponde a una regresión PraisWinsten, con corrección de error estándar en paneles.

Una vez introducidos estos ajustes, los resultados para el primer grupo de variables indican que el efecto de estas variables dentro de los países entre 1970 y 2014 es marginal. La inflación prácticamente no tiene ningún efecto sobre una transformación del Estado, lo que indica que el objetivo de esa política de ajuste era más destinado a una coyuntura específica. En el caso de la importancia que tiene en el período una mayor exposición al comercio internacional, tiene un efecto irrelevante en una reducción estatal como actor económico activo.

Los parámetros que presentan una mayor capacidad predictiva, el ingreso per cápita y el PIB, dejan de ser significativos un $90 \%$ y $91 \%$ de confianza, una vez aplicada la corrección PCSE, que señala como el parámetro más importante la reducción del gasto general del gobierno y su efecto sobre un aumento de la importancia del sector privado como actor económico. Un aumento en un punto en el gasto general del gobierno disminuye la importancia del sector privado en 0,01 puntos por año (Tabla 2) En la comparación con el modelo de efecto fijo, la aplicación de una regresión PCSE, disminuye la capacidad predictiva de los parámetros, pero a la vez disminuye el sesgo de los mismos. Asimismo, incrementó la bondad de ajuste del modelo. 


\section{Tabla 2: Modelo 1. Efecto de variables macroeconómicas sobre el papel del Estado en la economía 1970-2014}

\begin{tabular}{ccc} 
Variables & Efecto fijo & PCSE \\
\hline Gasto general del gobierno & $-0,0154^{* * *}$ & $-0,0140 * * *$ \\
(porcentaje PIB) & $(-4,30)$ & $(-3,47)$ \\
Inflación (deflactor PIB) & $-0,0000169$ & 0,0000071 \\
Comercio sobre PIB & $(-1,02)$ & $(0,83)$ \\
(porcentajePIB) & 0,000631 & 0,000286 \\
PIB (log) & $(0,65)$ & $(0,36)$ \\
& $1,084^{* * *}$ & 0,0716 \\
PIB per cápita (log) & $(16,26)$ & $(1,72)$ \\
& $-1,235 * * *$ & $-0,171$ \\
Constante & $(-10,99)$ & $(-1,65)$ \\
& $-13,48^{* * *}$ & $2,464 * * *$ \\
$\mathrm{R}^{2}$ & $(-14,47)$ & $(3,47)$ \\
Rho & 0,314 & 0,424 \\
$\mathrm{~N}$ & 0,939 & 0,919 \\
& 841 & 841 \\
& $*<0,05, * * \mathrm{p}<0,01, * * * \mathrm{p}<0,001$
\end{tabular}

En el caso del efecto de determinados patrones exportadores respecto a disminución de la importancia del Estado como actor económico (poseedor de activos), estos si bien presente un efecto positivo, este es muy bajo, dado que es un valor muy cercano a cero Es decir, los parámetros asociados indican que el aumento de un punto en la importancia de la renta de los recursos naturales, implica el aumento de un mayor protagonismo del Estado, pero de manera marginal, 0,002\% anual (pero a un $70 \%$ de confianza). En el caso la importancia de un sector industrial dinámico, su relevancia es aún menor, lo que implica en que la confianza de ese parámetro es de solo 10\% (Tabla 3, Modelo 2)

Para el caso de la presencia de los acuerdos comerciales indicados, la interpretación de los parámetros asociados, se observan niveles de confianza bastante alejados de un 95\%. En el caso de la presencia de un acuerdo con los Estados Unidos, un 70\%, mientras que respecto a la presencia del 
acuerdo GATT, el nivel de confianza es superior, 84\%. Tomando en cuenta ello, la presencia de este último tendría un mayor impacto en la reducción del papel económico del Estado, con un incremento de 0,05 puntos en la variable State. Estos datos, harían tomar con cierto cuidado los argumentos expuestos respecto a la efectividad de estos acuerdos (Tabla 3, Modelo 3).

Finalmente, se expone el modelo (4), que considera a todas las variables estudiadas, según las categorías prefijadas. Una primera comparación, señala que la bondad de ajuste del modelo no se incrementa demasiado, pasando a tener un $\mathrm{r}^{2}$ de 0,50 , pero con nueve predictores considerados. Al considerar el conjunto de variables, aumenta levemente el efecto de un menor gasto, donde el aumento de un punto anual de un punto en el gasto general del gobierno, tienen una disminución de 0,018 puntos en la importancia del sector privado en la economía y, por lo tanto, más relevancia del Estado.

El efecto de un mayor PIB, pasa a tener una importancia, aun cuando los parámetros son bastante parecidos. Sin embargo, al considerarlos en conjunto, el efecto del incremento del PIB $(\log )$ en una unidad, implica un aumento de 0,078 puntos en la disminución de la importancia del Estado como actor económico. En el caso del incremento del ingreso per cápita, el aumento en una unidad (log), implica una mayor importancia del Estado. Esta lectura indicaría que la relevancia del sector privado en la riqueza no tiene un efecto positivo. En ambos casos, los parámetros tienen una confianza del $95 \%$.

La renta obtenida de los recursos naturales tiene un efecto en el aumento de la importancia del Estado como actor económico. Ello se entendería por su mayor disponibilidad de recursos para gasto. No obstante, el impacto es pequeño, aun cuando hay un incremento de 2,,5 veces al comparar el tamaño de ambos parámetros y al ser incorporada en el conjunto de variables adquiere un nivel de confianza del 95\%.

La presencia de un acuerdo de comercio con los Estados Unidos toma una mayor importancia cuando es considerada dentro del conjunto de variables. Tomando en cuenta el tamaño de su parámetro, este se incrementa 2,14 veces, donde un tratado comercial vigente con la principal potencia hemisférica, tiene un incremento de 0,0993 puntos en una reducción de la importancia del Estado como actor económico. Ello 
confirmaría los argumentos que referirían a la política comercial en cuanto a su uso como instrumento de consolidación de un modelo económico.

\section{Tabla 3: Comparación de modelos 1970-2014. Modelos PCSE. Variable dependiente. Rol de Estado en la economía}

\begin{tabular}{|c|c|c|c|c|}
\hline Variables & Modelo 1 & Modelo 2 & Modelo 3 & Modelo 4 \\
\hline $\begin{array}{l}\text { Gasto general del gobierno } \\
\text { (porcentaje PIB) }\end{array}$ & $\begin{array}{c}-0,0140^{* * *} \\
(-3,47)\end{array}$ & - & - & $\begin{array}{c}-0,0177 * * * \\
(-3,63)\end{array}$ \\
\hline Inflación (deflactor PIB) & $\begin{array}{l}0,00000710 \\
(0,83)\end{array}$ & - & - & $\begin{array}{l}0,0000104 \\
(1,07)\end{array}$ \\
\hline $\begin{array}{l}\text { Comercio sobre PIB } \\
\text { (porcentaje PIB) }\end{array}$ & $\begin{array}{l}0,000286 \\
(0,36)\end{array}$ & - & - & $\begin{array}{c}0,00144 \\
(1,67)\end{array}$ \\
\hline PIB (log) & $\begin{array}{c}0,0716 \\
(1,72)\end{array}$ & - & - & $\begin{array}{c}0,0777 * \\
(2,05)\end{array}$ \\
\hline PIB per cápita (log) & $\begin{array}{l}-0,171 \\
(-1,65)\end{array}$ & - & - & $\begin{array}{c}-0,202^{*} \\
(-2,15)\end{array}$ \\
\hline $\begin{array}{l}\text { Renta de los recursos naturales } \\
\text { (porcentaje PIB) }\end{array}$ & - & $\begin{array}{c}-0,00178 \\
(-1,03)\end{array}$ & - & $\begin{array}{c}-0,00447 * \\
(-2,42)\end{array}$ \\
\hline $\begin{array}{l}\text { Exportaciones manufacturas } \\
\text { (porcentaje de exportaciones) }\end{array}$ & - & $\begin{array}{c}-0,0000229 \\
(-0,04)\end{array}$ & - & $\begin{array}{c}0,000296 \\
(0,45)\end{array}$ \\
\hline $\begin{array}{l}\text { Presencia acuerdo con EE.UU } \\
\text { (1) }\end{array}$ & - & - & $\begin{array}{c}0,0452 \\
(1,04)\end{array}$ & $\begin{array}{c}0,0993^{*} \\
(2,00)\end{array}$ \\
\hline Presencia de acuerdo GATT (1) & - & - & $\begin{array}{c}0,0464 * * \\
(1,42)\end{array}$ & $\begin{array}{c}0,0398 \\
(1,13)\end{array}$ \\
\hline Constante & $\begin{array}{c}2,464 * * * \\
(3,47)\end{array}$ & $\begin{array}{c}2,636^{* * *} \\
(31,94)\end{array}$ & $\begin{array}{c}2,598^{* * *} \\
(30,08)\end{array}$ & $\begin{array}{c}2,528^{* * *} \\
(4,00)\end{array}$ \\
\hline $\mathrm{R}^{2}$ & 0,424 & 0,401 & 0,312 & 0,503 \\
\hline Rho & 0,919 & 0,932 & 0,943 & 0,889 \\
\hline $\mathrm{N}$ & 841 & 825 & 886 & 782 \\
\hline
\end{tabular}

\section{DISCUSIÓN Y CONCLUSIONES}

En la evolución hacia una mayor importancia del sector privado en la economía, que es una característica que define a la variable dependiente en el período señalado, aquellos predictores relacionados con el comercio internacional no tienen relevancia en el período 1970-2014. El aumento del comercio en relación al PIB tiene un efecto en aumentar la importancia del sector privado en la economía en desmedro del Estado, pero marginalmente. 
Ello sugiere que el patrón de comercio de los países considerados es independiente respecto de los cambios que se producen en el rol estatal. Este elemento se confirmaría con el efecto que tiene la dependencia de los recursos naturales, respecto a la renta obtenida, por parte de los países. Esta caratcerística de los países latinomaericanos se observa también en la revisión biliográfica.

Sí e es relevante que esta disminución de los niveles de gasto gubernamentales tienen un efecto a considerar en una mayor importancia del sector privado en la economía. Ello dice relación con que el control del déficit público no es solo una medida destinada a un evento espécifico, como si se observa en el caso de medidas destinadas al control inflacionario, sino que precisamente un bajo gasto es una característica de países cuyos gobiernos reducen el papel de sus estados en sus respectivos ordenamientos económicos. En ese sentido la revisión bibliográfica, detallada en el primer apartado confirmaría este argumento.

Este punto permite introducir un matiz respecto al papel que tendría el comercio en la disminución del rol estatal en la economía, donde el punto central es que una orientación liberalizadora del mismo. En ese sentido, esa característica no aparece clara al examinar el efecto de los patrones de comercio. En el caso del acuerdo GATT, que sigue una lógica de limitar la protección, tiene un efecto bastante limitado. En cambio, en el caso de la presencia de un ACR con los Estados Unidos, que daría cuenta de una orientación liberalizadora, tiene un impacto mayor en la disminución del papel del Estado en la economía, a un 95\% de confianza.

En ese sentido, la hipótesis de trabajo que se planteó como orientadora del presente artículo, (las variables relacionadas con el comercio tienen un efecto sobre una disminución en el rol del Estado en la economía entre 1970 y 2014) no puede responderse de una manera clara. No obstante, hay indicios que estas variables sí pueden tener algún efecto si se logra considerar su carácter liberalizador. Esta se caracterizaría al estimar el impacto sobre la variable State, por parte de la mayor orientación hacia el libre mercado de un país, que serían los resultados obtenidos por cada uno de ellos el Índice de Libertad Económica ${ }^{6}$ (IEF) de la Heritage Foundation

6 Este índice se elabora en una escala de 1 a 100, donde este último valor indica una adhesión plena a la libertad económica por parte del país evaluado. Los valores iguales o superiores a 80 puntos, indican la presencia de una economía libre, mientras que 
y del Wall Street Journal, disponibles desde 1995 (Heritage Foundation y Wall Street Journal 2014).

El resultado indica que por cada aumento en el IEF, la mayor importancia del sector privado en desmedro del Estado se incrementa anualmente en 0,019 puntos, considerando que este ocurre al interior de las entidades del panel. Este parámetro es significativo a un $\mathrm{p}<0000$. La bondad de ajuste que presenta ese modelo es de un 0,75 , la que es bastante elevada considerando que se trata de solo una. Su limitación, no obstante, que este resultado es válido solo para un período posterior a 1995, lo que impediría observar los efectos en un largo plazo mayor, como lo propuesto en el presente documento. En ese sentido, como preguntas abiertas queda estudiar el efecto de la apertura comercial respecto a la transformación del estado como actor en la economía, que tenga a su vez el objetivo de liberalizar.

sobre 60 se considera a la economía como "moderadamente libre". En el caso de los inferiores a 80 , representan a países que serían represivos de la libertad económica (Heritage Foundation y Wall Street Journal 2014). 


\section{REFERENCIAS}

Allee, T. y Elsig, M. (2016). Why Do Some International Institutions Contain Strong Dispute Settlement Provisions? New Evidence from Preferential Trade Agreements. The Review of International Organizations, 11 (1), 89-120.

Allison, P. D. (2009). Fixed Effects Regression Models. Londres: SAGE.

Anderson, R. D., Pelletier, P., Osei-Lah, K. y Müller, A. C. (2011). Assessing the Value of Future Accessions to the WTO Agreement on Gouvenement Procurement (GPA): Some New Data Sources, Provisional Estimates, and an Evaluative Framework for Individual WTO Members Considering Accession: Ginebra: WTO Staff Working Paper.

Baccini, L., Dür, A., Elsig, M. y Milewicz, K. (2011). The Design of Preferential Trade Agreements: A New Dataset in the Making. Ginebra: WTO Staff Working Paper.

Baccini, L., y Urpelainen, J. (2014). International Institutions and Domestic Politics: Can Preferential Trading Agreements Help Leaders Promote Economic Reform? The Journal of Politics, 76 (1), 195-214.

Baer, W. (1972). Import Substitution and Industrialization in Latin America: Experiences and Interpretations. Latin American Research Review, 7 (1), 95-122.

Banco Mundial. (2014). World Development Indicators. Disponible en http://databank.worldbank.org/data/home.aspx [01-06-2016.]

Beck, N. (2008). Time-Series-Cross-Section Methods. En BoxSteffensmeier, J. M., Brady, H. y Collier, D. (Eds.), Oxford Handbook of Political Methodology. Oxford: Oxford University Press.

Beck, N. y Katz, J. N. (1995). What to Do (and not to Do) with TimeSeries Cross-Section Data. American Political Science Review, 89 (3), 634-647. 
Bell, A. y Jones, K. (2015). Explaining Fixed Effects: Random Effects Modeling of Time-Series Cross-Sectional and Panel Data. Political Science Research and Methods, 3 (1), 133-153.

Bresser Pereira, L. C., Maravall, J. M. y Przeworski, A. (1995). Las Reformas Económicas en las Nuevas Democracias. Un Enfoque Socialdemocrata. Madrid: Alianza Editorial.

Cardoso, F. H. y Falleto, E. (1969). Dependencia y Desarrollo en América Latina. Un Ensayo de Interpretación Sociológica. Buenos Aires: Siglo XXI.

Coppedge, M. et al. (2016). Varieties of Democracy Codebook v6.. Gothenburg: University of Gothenbourg - University of Notre-Dame.

Crouch, C. (2009). Privatised Keynesianism: An Unacknowledged Policy Regime. The British Journal of Politics \& International Relations, 11 (3), 382-399.

Cambridge: Polity.

(2011). The Strange Non-Death of Neo-Liberalism. . (2013). Making Capitalism Fit for Society: Cambridge:

Polity.

De Melo, M., Denizer, C., Gelb, A. y Tenev, S. (2001). Circumstance and Choice: The Role of Initial Conditions and Policies in Transition Economies. The World Bank Economic Review, 15 (1), 1-31.

Dür, A., Baccini, L. y Elsig, M. (2014). The Design of International Trade Agreements: Introducing a New Dataset. The Review of International Organizations, 9 (3), 353-375.

Elsig, M. (2007). The EU's Choice of Regulatory Venues for Trade Negotiations: A Tale of Agency Power? JCMS: Journal of Common Market Studies, 45 (4), 927-948.

Evans, P. y Sewell, W. (2013). Policy Regimes, International Regimes and Social Effects. En Hall, P. A. y Lamont, M. (Eds.), Social Resilience In 
the Neoliberal Nueva York: Cambridge University Press.

Ffrench-Davis, R. (1973). Politicas Económicas en Chile. 1952-1970. Santiago: CIEPLAN, Ediciones Nueva Universidad.

Gathii, J. T. (2011). The Neoliberal Turn in Regional Trade Agreements. Washington Law Review, 86 (3), 421-474.

Goode, W. (2007). Dictionary of Trade Policy Terms. Cambridge: Cambridge University Press.

Haggard, S., y Kaufman, R. R. (1997). The Political Economy of Democratic Transitions. Comparative Politics, 29 (3), 263-283.

Henrik, H., Mavroidis, P. C. y Sapir, A. (2009). Beyond the WTO? An Anatomy of EU and US Preferential Trade Agreements. Bruselas: Bruegel Blue Print Series.

Heritage Foundation, y Wall Street Journal. (2014). Index of Economic Freedom. Disponible en http://www.heritage.org/index/ [01-062016].

Hirschman, A. O. (1968). The Political Economy of Import-Substituting Industrialization in Latin America. The Quarterly Journal of Economics, $82(1), 1-32$.

Love, J. L. (1990). The Origins of Dependency Analysis. Journal of Latin American Studies, 22 (1-2), 143-168.

Low, C., Elms, D. y Low, P. (2012). Trans-Pacific Partnership: The Quest for a 21st Century Trade Agreement: Cambridge: Cambridge University Press.

Mahoney, J. y Goertz, G. (2006). A Tale of Two Cultures: Contrasting Quantitative and Qualitative Research. Political Analysis, 14 (3), 227249.

Mansfield, E. D., y Milner, H. V. (Eds.). (2012). A Political Economy Theory of International Trade Agreements. En Mansfield, E. D. 
y Milner, H. V. (Eds.), Votes, Vetoes, and the Political Economy of International Trade Agreements: Princeton: Princeton University Press.

Mansfield, E. D. y Milner, H. V. (2012). Votes, Vetoes, and the Political Economy of International Trade Agreements: Princeton: Princeton University Press.

Murillo, M. V. (2001). Labor Unions, Partisan Coalitions, and Market Reforms in Latin America, Nueva York: Cambridge University Press.

Organización de Estados Americanos (OEA). (2015). Sistema de Información de Comercio Exterior. Disponible en http://www.sice. oas.org/agreements_e.asp [01-06-2016].

Organización Mundial del Trabajo (OMC). (2011). La OMC y los Acuerdos Comerciales Preferenciales: De La Coexistencia a la Coherencia. En OMC (Ed.), Informe sobre el Comercio Mundial. Ginebra: Organización Mundial del Trabajo.

. (2015). Regional Trade Agreements Information System (RTA-IS). Disponible en http://rtais.wto.org/UI/ PublicMaintainRTAHome.aspx [01-06-2016].

Organización Mundial del Comercio (OMC), International Trade Center y Conferencia de las Naciones Unidas sobre Comercio y Desarrollo (UNCTAD). (2013). World Tariff Profiles. Ginebra: OMC, ITC, UNCTAD.

Palma, J. G. (1978). Dependency: A Formal Theory of Underdevelopment or a Methodology for the Analysis of Concrete Situations of Underdevelopment? World Development, 6 (7-8), 881-924.

Plümper, T., Troeger, V. E. y Manow, P. (2005). Panel Data Analysis in Comparative Politics: Linking Method to Theory. European Journal of Political Research, 44 (2), 327-354.

Prebisch, R. (1986). El desarrollo Económico de la América Latina y Algunos de sus Principales Problemas. Desarrollo económico, 26 (103), 479-502. 
Przeworski, A. (1991). Democracy and the Market: Political and Economic Reforms in Eastern Europe and Latin America: Nueva York: Cambridge University Press.

Schmidt, V. y Tatcher, M. (Eds.). (2013). Resilient Liberalism in Europe's Political Economy. (2013). (V. A. Schmidt y M. Thatcher Eds.). Nueva York: Cambridge University Press.

Rodrik, D. (2000). Trade Policy Reform as Institutional Reform. Washington: Banco Interamericano de Dessarrollo,

Schroeder, L. D., Sjoquist, D. L. y Stephan, P. E. (1986). Understanding Regression Analysis (Vol. 57). Newbury Park: SAGE.

StataCorp. (2013). Stata. Longitudinal -Data/Panel Data, Reference Manual. Release 13. College Station: Stata Press.

Streeck, W. (2012). How to Study Contemporary Capitalism? European Journal of Sociology, 53 (1), 1-28.

Urquidi, V. H. (2005). Otro Siglo Perdido. Las Politicas de Desarrollo en América Latina. México: Fondo de Cultura Económica.

Recibido: 02-05-2016

Aceptación de la versión final: 24-06-2016 